\title{
BMJ Open What are the health outcomes of trans and gender diverse young people in Australia? Study protocol for the Trans20 longitudinal cohort study
}

\author{
Michelle Anne Tollit (D) , , ,2,3 Carmen C Pace, ${ }^{1,2,4}$ Michelle Telfer, ${ }^{1,2,4}$ \\ Monsurul Hoq (D) , Janet Bryson (D) , ${ }^{1}$ Nicholas Fulkoski, ${ }^{1}$ Charlie Cooper, ${ }^{1}$ \\ Ken C Pang (1) 1,2,4,6
}

To cite: Tollit MA, Pace CC, Telfer M, et al. What are the health outcomes of trans and gender diverse young people in Australia? Study protocol for the Trans20 longitudinal cohort study. BMJ Open 2019;9:e032151. doi:10.1136/ bmjopen-2019-032151

- Prepublication history for this paper is available online. To view these files, please visit the journal online (http://dx.doi. org/10.1136/bmjopen-2019032151).

Received 05 June 2019 Revised 19 September 2019 Accepted 23 September 2019

Check for updates

(C) Author(s) (or their employer(s)) 2019. Re-use permitted under CC BY-NC. No commercial re-use. See rights and permissions. Published by BMJ.

For numbered affiliations see end of article.

Correspondence to

Dr Ken C Pang;

ken.pang@mcri.edu.au

\section{ABSTRACT}

Introduction Being transgender is frequently accompanied by gender dysphoria, which often coexists with mental health concerns. Increased referrals of transgender and gender diverse (TGD) youth to gender clinics have been observed in many countries. Nevertheless, there are limited empirical data on the presentation and outcomes of these patients, and there is an urgent need for more evidence to ensure optimal medical and psychosocial interventions. Here, we describe Trans20, a longitudinal study of TGD patients attending a multidisciplinary paediatric gender service in Melbourne, Australia. Trans20 aims to understand the demographic and clinical characteristics of these patients, to document the natural history of gender diversity presenting in childhood and to investigate long-term outcomes for those receiving interventions.

Methods and analysis Trans20 is a prospective cohort study based on children and adolescents first seen at the Royal Children's Hospital Gender Service (RCHGS) between February 2017 and February 2020. Current estimates indicate the final sample size will be approximately 600 . Patients and their parents complete online questionnaires prior to the first appointment with RCHGS and regularly thereafter as part of routine clinical care. On discharge from RCHGS, patients are invited to continue undertaking questionnaires biennially. In this way, a naturally forming cohort study has been created. The primary outcomes include gender dysphoria, physical and mental health, schooling, family functioning and quality of life. Subgroup analyses based on factors such as gender identity, birth-assigned sex and treatment received will be performed using bivariate and multivariate modelling as appropriate, and relevant statistical methods will be applied for the repeated measures over time.

Ethics and dissemination The Royal Children's Hospital Human Research Ethics Committee approved this study (\#36323). Findings from Trans20 will have translational impact by informing future treatment guidelines and gender affirming healthcare practices and will be disseminated through conferences and peer reviewed journals.

\section{INTRODUCTION}

In recent times, there has been an increase in the number of individuals identifying
Strengths and limitations of this study

- Multiple domains including gender, mental health, physical health, schooling, family functioning and quality of life will be prospectively documented in a large cohort of transgender and gender diverse (TGD) children and adolescents.

- Trans20 will represent one of the largest and most comprehensive longitudinal studies of TGD young people in the world, with potential to make a significant contribution to better understanding and improving the health of TGD children and adolescents globally.

- The long-term follow-up of prepubescent children will allow examination of the natural history of gender diversity from an early age in a clinically referred population, enabling clinicians to provide accurate prognostic information to patients and families and therefore assist decision-making around social and legal transition for TGD young people.

- Due to ethical reasons, it is not possible to incorporate an untreated control group in the Trans20 study design; this will limit the potential to draw direct conclusions about the effectiveness of interventions but, where possible, measures with population-based data are used in Trans20 to compare outcomes of TGD youth with those of the general population.

as transgender ${ }^{1}$ and those seeking gender affirming healthcare. ${ }^{2}$ This rise may in part be related to increased social acceptance of gender diversity and the growing recognition that being transgender is part of the continuum of gender diversity. Despite these shifts in societal attitudes, being transgender is frequently accompanied by gender dysphoria (GD). GD is the distress that arises when a person's birth-assigned sex does not match their gender identity. GD experienced in childhood and adolescence can be particularly challenging as young people are navigating a range of physical, social and 
emotional changes during this critical developmental period. Serious psychiatric disorders are very common, with rates of self-reported depression and anxiety diagnoses in transgender and gender diverse (TGD) young people in Australia as high as $75 \%$ and $72 \%$, respectively, and $80 \%$ reporting ever self-harming and $48 \%$ ever attempting suicide. ${ }^{3}$ Experiences of bullying, physical assault, discrimination and social exclusion are also common for transgender individuals, ${ }^{3-6}$ and these experiences are likely to contribute to poor mental health.

Accompanying the rise in reported prevalence of TGD young people has been a rapid increase in referrals to specialist gender clinics across the Western world. ${ }^{7-10}$ Many TGD children and adolescents and their families seek help from healthcare professionals requesting support, advice and gender affirming psychosocial and medical treatments. Clinical guidelines ${ }^{11-13}$ promote a multidisciplinary approach to address the complex biopsychosocial needs of this group, and treatment is ideally tailored to a young person's developmental stage and individual needs. Psychosocial support is central to assist the young person and their parents to understand and explore their gender identity and, where appropriate, help facilitate social transition, which may involve adoption of gender-affirming hairstyles, clothing, names and pronouns. For children and adolescents, psychological support can also be an important intervention for treating coexisting mental health issues, while helping to navigate the relational, social and personal challenges associated with their gender identity. For children who have not reached puberty-some of whom present with significant GD as young as age three-medical intervention is not warranted, but for older children and adolescents, medical interventions may serve an important role alongside psychosocial support and can take several forms. First, medications known as GnRH analogues ('puberty blockers') can help prevent the development of undesired physical changes during puberty, which can trigger and/or exacerbate GD. Second, gender affirming hormones, namely oestrogen and testosterone, can help promote physical changes congruent with the young person's gender identity. Thirdly, surgical procedures, such as chest reconstructive surgery for transmasculine individuals ('top surgery'), are performed on adolescents in some centres ${ }^{14}{ }^{15}$ while genital surgery is generally only advised after the age of majority. ${ }^{11} 12$

Specific healthcare for TGD children and adolescents-including the use of medical interventions-is relatively new, having commenced only in the past two decades. Consequently, there is a need for more empirical data to inform best practice in important areas such as risk and protective factors and the long-term safety and outcomes of medical interventions. ${ }^{12} 1617$ Another key area for which stronger evidence is required relates to the natural history of gender diversity. While some parents of gender diverse youth report noticing signs of gender non-conforming behaviour from as young as age two,${ }^{18}$ not all gender diverse children develop a transgender identity. Published literature reports that $45 \%-88 \%$ of children with gender concerns in childhood go on to identify with their birth-assigned sex in adolescence and adulthood ${ }^{19-23}$ indicating that only some of these children report a transgender identity when older. A number of methodological, interpretative and ethical concerns have been raised about these studies, ${ }^{19}$ and they are now thought to underestimate the persistence of TGD identities from childhood to adolescence. Given this uncertainty, there is a clear need to better understand these trajectories in order to inform and guide decision making in this area for young people and their families.

Various research groups around the world have been attempting to fill these important knowledge gaps via longitudinal cohort studies of TGD children and adolescents. The longest running of these has been based at VU University Medical Centre in the Netherlands, which pioneered the use of medical interventions for TGD youth and has provided much of the currently available empirical data in the field. ${ }^{24}$ More recently, an NIH-funded study commenced in the USA and is aiming to follow 280 transgender youth over 5 years. ${ }^{25}$ The study is recruiting across four separate clinical sites, each of which have their own treatment protocols and practices. There is a clear need for additional international cohorts to sit alongside these established studies.

This paper describes the creation of a new longitudinal study of TGD children and adolescents, known as Trans20. Following a large cohort of patients who have attended the state-wide Royal Children's Hospital Gender Service (RCHGS) in Melbourne, Australia, Trans20 is designed to synthesise prospectively gathered clinical data collected from patients during their time with and after they leave the RCHGS. The RCHGS employs a consistent assessment and treatment approach to its trans healthcare, meaning that Trans20 is ideally placed to avoid the difficulties inherent in studies that recruit across multiple sites where treatment approaches may vary.

In the context of this background, the Trans20 study primarily aims to:

i. Describe the demographic and clinical characteristics of TGD young people who attend healthcare services for gender-related concerns.

ii. Identify clinical outcomes following different types of gender-affirming interventions (both psychosocial and medical).

iii. Identify risk and protective factors associated with physical and mental health outcomes of TGD children and adolescents.

iv. Characterise the natural history of TGD young people, including those who differ in their presentations.

Trans20 seeks to contribute to the evidence underpinning healthcare for TGD young people, and thus inform prognosis and improve clinical management and decision making. 
Legend

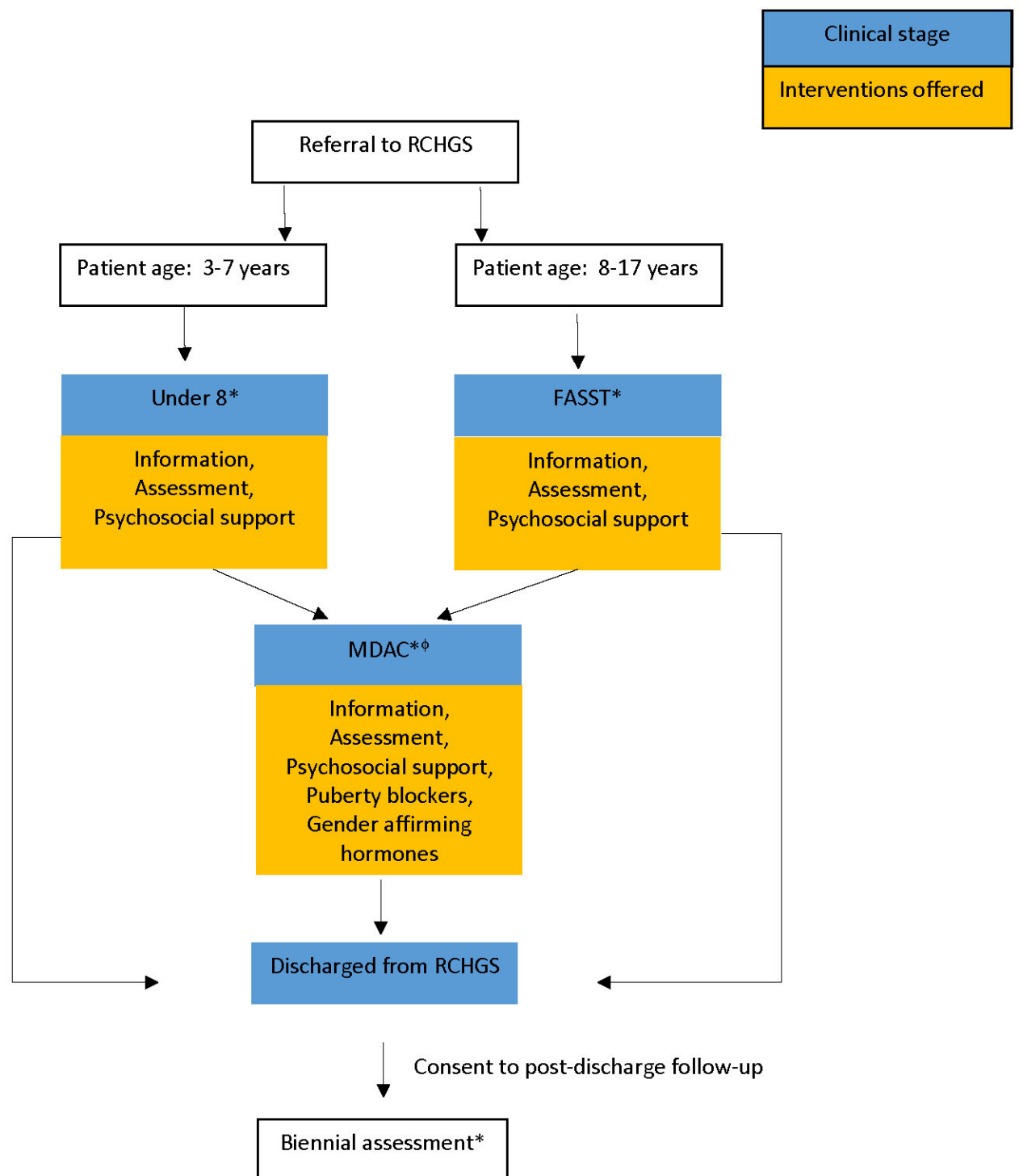

Figure 1 Clinical pathway at the RCHGS and integrated questionnaire administration. *Indicates data collection timepoint for Trans20. ФFollowing initial attendance at MDAC, questionnaires are repeated on an annual basis. FASST, First Assessment Single Session Triage; MDAC, Multidisciplinary Assessment Clinic; RCHGS, Royal Children's Hospital Gender Service.

\section{METHODS AND ANALYSIS}

\section{Study design}

Trans20 is a prospective, longitudinal cohort study, with a sample comprising patients aged 3-17 years when first attending the RCHGS between February 2017 and February 2020. Online questionnaires are routinely administered as part of standard clinical care at RCHGS and are completed by patients and a nominated parent/ primary caregiver prior to the first appointment with the service and then at regular periods thereafter (figure 1). Some questionnaires are also administered by clinicians during the patients' clinical consultations, and these are referred to as 'in-clinic questionnaires' hereafter. Patients who leave the service are invited to continue undertaking questionnaires biennially. In this way, a naturally forming cohort study has been created, and our intention is to continue follow-up over a 20 -year period.

\section{Study setting}

This study is based at the RCHGS in Melbourne, Australia. The RCHGS provides publicly funded assessment and gender affirming care to TGD young people throughout the state of Victoria. RCHGS staff come from a range of clinical specialities and disciplines including psychology, psychiatry, paediatrics, endocrinology, gynaecology, speech therapy and nursing. With approximately $250 \mathrm{new}$ referrals each year, RCHGS is the largest multidisciplinary gender service for children and adolescents in Australia. Young people up to age 17 years who reside in Victoria and have concerns regarding their gender identity can be referred to RCHGS by their general practitioner. Patients 
subsequently enter the service via one of two pathways: the Under 8 clinic for those aged 7 years and below, or the First Assessment Single Session Triage (FASST) clinic for those aged 8 years and older. Patients attending the Under 8 clinic are assigned a mental health clinician for initial assessment and support and those wishing to undertake a medical transition are later referred to a member of the paediatric team prior to onset of puberty. Patients attending the FASST clinic see a Clinical Nurse Consultant or an Adolescent Medicine fellow (doctor undergoing advanced paediatric training) for initial assessment and support; those who wish to receive further psychosocial support and/or medical intervention are subsequently seen in the Multidisciplinary Assessment Clinic (MDAC) (figure 1). Regardless of which pathway a patient enters, they and their parents undertake an assessment which includes the completion of standardised questionnaires prior to their first appointment, and these assessments are repeated at regular ( 12 monthly) intervals as patients continue to attend the service.

\section{Participants and eligibility}

To be eligible for inclusion in Trans20, participants need to have attended an initial appointment with the RCHGS between February 2017 and February 2020, have completed at least one of the baseline questionnaires (ie, patient questionnaire, parent questionnaire, or in-clinic questionnaire) and speak sufficient English to complete the questionnaires. Since patients can be referred to the RCHGS at any age before 18 years, participants are expected to range in age from 3 to 17 years at study entry. Data from the first 2 years of the study indicate that on the day of first appointment with the RCHGS, the large majority of patients $(75.3 \%)$ were aged 12 years or older, $18.6 \%$ were aged $6-11$ years and $6.1 \%$ were aged 5 years or younger. Based on those meeting eligibility for involvement in Trans20 in the first 2 years, it is expected that the Trans20 cohort will comprise approximately 600 participants over the 3-year enrolment period.

\section{Ethics, recruitment and consent}

The Royal Children's Hospital Human Research Ethics Committee (HREC) granted approval for the conduct of this study (\#36323). As previously outlined, questionnaires are completed by patients and parents as part of routine clinical care at RCHGS, and this information will be analysed under a clinical audit framework. As Trans20 is designed to prospectively collect data from patients beyond their time with the RCHGS, long-term follow-up of patients who leave the RCHGS requires specific consent. Consent is obtained using a multistep procedure, from parents for those aged under 18 years and from young people themselves once they are aged 18 years or older. At the time of undertaking assessments with the RCHGS, permission to contact patients and their families about the Trans20 study in the future is prospectively collected. Following discharge, those who have provided consent to be contacted are then invited to continue to undertake assessments. For patients who have left the service and are under 18 years, an email with parent and participant information statements as well as questionnaire links are sent to the nominated parent, who are asked to pass the relevant documentation to their child if they consent to them being involved. Patients who have left the service and are 18 years or older are sent these directly via email. Under each of these scenarios, completion of the online questionnaires provides implied consent. Consent to contact treating clinicians to obtain physical health information will also be sought for those who no longer attend the RCHGS.

\section{Data collection and storage}

Parent and patient questionnaires are administered via LimeSurvey, an online, open source, survey web application, ${ }^{26}$ supported by the RCH for clinical use. Separate parent and patient questionnaire links are sent to a parent email address and parents and patients complete the questionnaires online. Questionnaires are administered approximately 1 month prior to patients' initial appointment with the service and then at approximately 12 month intervals throughout their RCHGS episode of care. Information on community supports are provided at the end of all questionnaires. Participants (who no longer attend the RCHGS) who disclose information that raises concern about a significant risk of harm will be contacted by project staff to provide additional support. This may include: discussing additional support services, encouraging the participant to contact their general practitioner and/or access existing supports and referring the participant to external supports (including mental health triaging services) where appropriate. The information statements that accompany the surveys, advises parents and participants that they may be contacted for this purpose. If individuals remain upset after completing questionnaires, they are also advised to call the RCHGS to help organise support.

In-clinic questionnaires-which cover topics such as drug use, sexual health, self-harm and suicidality-are asked directly of patients at an appropriate age by clinicians during their appointments and responses are later entered into LimeSurvey. These questions are asked by clinicians during appointments to ensure timely and appropriate follow-up if required. Having been introduced by their clinicians, these questions are subsequently asked of patients in their online follow-up questionnaires, with clinicians applying appropriate follow-up for current patients as deemed clinically relevant.

Parent, patient and in-clinic questionnaire responses (and scored summary data where relevant) are uploaded to patients' electronic medical record (EMR) and the RCHGS Clinical Database (DRN \#DB089). Thus, these are available to their treating team to help guide assessment and treatment. For those who consent to being part of Trans20 following discharge from the RCHGS, follow-up questionnaires are administered via LimeSurvey and stored in the RCHGS Clinical Database. For 
those discharged from RCHGS, a $\$ 20$ gift voucher will be offered as a sign of appreciation and as reimbursement for the time spent completing the surveys.

Data from this study are stored securely on RCH servers in password-protected files that can only be accessed by study personnel. Data will be stored for 7 years post study closure, or until the youngest participant turns 25 years old and will then be securely destroyed. Data will be stored in a reidentifiable format, with participants given a unique, reidentifiable code that maps to identifying information, including hospital record number.

\section{Measures}

Trans20 questionnaire measures were selected to provide information relevant to the assessment and treatment of GD and related comorbidities. Measures span multiple domains including gender, mental health, education, quality of life, parental well-being, family functioning as well as experiences of care at RCHGS. In addition to measuring core gender-related and mental health outcomes, broader indicators of health and well-being were also included to holistically and comprehensively assess multifaceted functioning (including at school and within the family), which may be related to gender and mental health outcomes in this group. Where possible, measures with strong psychometric properties and good reliability and validity were chosen. Some measures are appropriate only for specific age groups or birth-assigned sex, and the online questionnaires are tailored accordingly. A full list of Trans20 questionnaire measures is provided in table 1 , and each measure-grouped by domain-is described in further detail below.

\section{Demographics and past history}

Standard demographic data and information related to the young person's medical and developmental history are routinely collected in the questionnaires. These items were developed by the study investigators; some demographic questions relating to English proficiency, Indigenous status and religious affiliation were based on standards recommended by the Australian Bureau of Statistics (ABS) ${ }^{27}$ From age 18 years, individuals are asked questions about education and employment based on ABS Census questions. ${ }^{28}$

\section{Gender}

Different aspects of gender identity are assessed using various complementary tools including the Gender Identity Questionnaire, ${ }^{29}$ Gender Preoccupation and Stability Questionnaire $^{30}$ and the Gender Slider. The Gender Slider, developed by the Trans20 project team in consultation with the RCHGS, is a visual analogue scale to help patients describe their gender identity. This tool consists of two continuous scales, male and female, where individuals move the slider on each scale to reflect their gender identity, from 'not at all' to 'completely'. The Body Image Scale ${ }^{31}$ provides information on body dissatisfaction. An item developed by the Trans 20 team is used to measure extent of GD. The Social Transition Questionnaire (developed by the Trans20 team) assesses the extent to which an individual has socially transitioned. The Gender Voice Questionnaire (adapted from the Transsexual Voice Questionnaire $^{32}{ }^{33}$ ) and the Chest Dysphoria Scale (administered to birth-assigned females) ${ }^{15}$ provide information on voice dysphoria and chest dysphoria, respectively. In combination, these tools enable the examination of shifts in gender identity, expression and dysphoria over time. Additional questions devised by the project team about the effects of hormonal treatment are asked of those who have been treated with hormones.

\section{Mental health}

Mental health is assessed longitudinally using wellvalidated measures chosen for their common use in this population. The Child Behavior Checklist (CBCL) and Youth Self Report ${ }^{34} 35$ assess behavioural and emotional problems across broad domains including: anxious/ depressed, withdrawn/depressed, somatic complaints, social problems, thought problems, attention problems, rule-breaking behaviour and aggressive behaviour. The Short Mood and Feelings Questionnaire ${ }^{36}$ and Spence Children's Anxiety Scale ${ }^{37-41}$ focus on depressive and anxiety symptomatology, respectively. In young adulthood, the DASS21 ${ }^{42}$ is used to measure depression, anxiety and stress while social phobia is measured using the Social Phobia Scale. ${ }^{43}$ The Gender Minority Stress and Resilience Measure (community connectedness and pride scales) ${ }^{44}$ and the Brief Resilience Scale ${ }^{45}$ measure resilience. Assessment of self-harm is based on an established self-harm questionnaire, ${ }^{46}$ and the ColumbiaSuicide Severity Rating Scale ${ }^{47}$ assesses suicidality. A modified version of the Branched Eating Disorders Test ${ }^{48}$ is administered to assess disordered eating. Given the frequent co-occurrence of autism in gender diverse individuals, ${ }^{49}$ the Social Responsiveness Scale- $2^{50}$ is used to screen for features of autism at the initial assessment.

\section{Education/school functioning}

Trans20 examines a range of educational outcomes over time. Academic achievement is measured using relevant questions within the CBCL. ${ }^{34}$ School connectedness is assessed using the Psychological Sense of School Membership survey. ${ }^{51}$ School absenteeism is determined using modified questions from the Longitudinal Study of Australian Children. ${ }^{52}$ Finally, bullying is evaluated using the Gatehouse Bullying Scale ${ }^{53}$ and supplemented with specific items inquiring about cyberbullying. Young adults are asked about experiences of discrimination based on questions from the Victorian Population Health Survey. ${ }^{54}$

\section{Quality of life and family functioning}

The Child Health Utility 9D (CHU 9D) $)^{55} 56$ and the Assessment of Quality of Life Instrument (AQoL-4D) $)^{57} 58$ are administered to determine how GD and its associated difficulties impact on child and parental quality of life, respectively. These tools are generic quality of life 


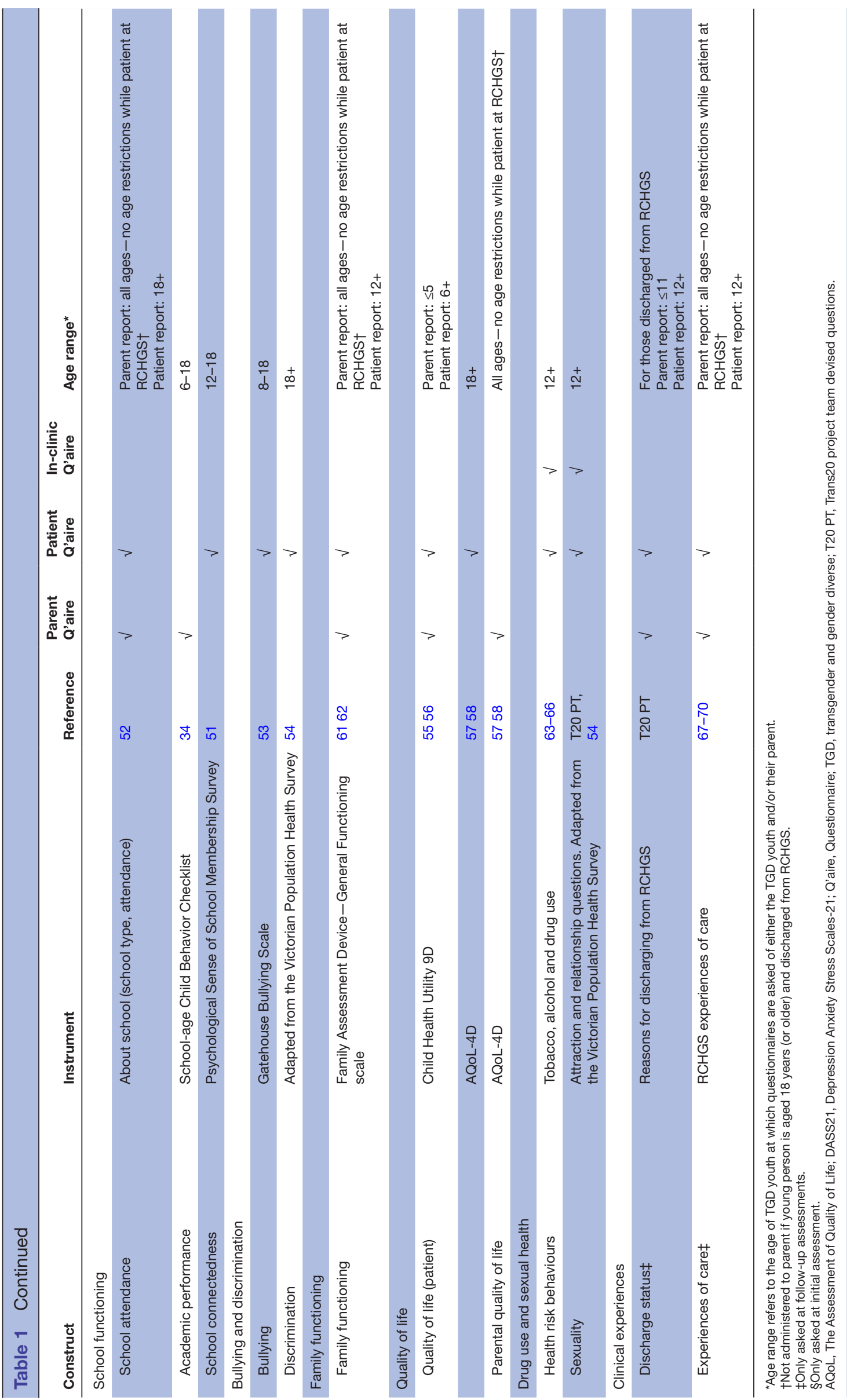

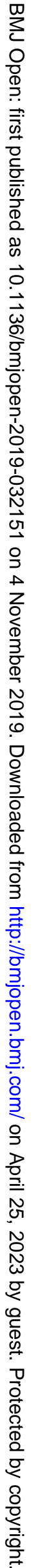


measures used frequently in health economic assessments of disease impact. Australian population-based norms and utility weights exist for the AQoL-4D and CHU-9D, respectively. ${ }^{5960}$ Family functioning is measured using the General Functioning 12-item subscale of The McMaster Family Assessment Device. ${ }^{6162}$

\section{Drug use and sexual health}

Adolescents and young adults are asked about current and lifetime alcohol, cigarette and illegal drug use, using questions adapted from the Communities That Care survey $^{63-65}$ and the Childhood to Adolescence Transition Study ${ }^{66}$ while sexual health information (including attraction, relationship status, current sexual activity) is gathered via items developed by the Trans20 team and items based on the Victorian Population Health Survey. ${ }^{54}$

\section{Experiences of care}

Young people (aged 12 and older) and all parents are asked about experiences of care at the RCHGS, using a questionnaire adapted from those in use at adult gender services in the USA, UK and Australia. ${ }^{67-70}$ Following discharge from the RCHGS, participants are asked the reason for discharge.

\section{Physiology}

To determine the physical effects of puberty blockers and gender affirming hormones, RCHGS clinicians routinely monitor a variety of physiological parameters, including height, weight, body mass index, blood pressure, bone mineral density, luteinising hormone, follicle stimulating hormone, testosterone, oestrogen, liver function, haemoglobin, serum cholesterol and haemoglobin A1c at regular intervals consistent with the Australian Standards of Care and Treatment Guidelines for Trans and Gender Diverse Children and Adolescents. ${ }^{13}$ These data are stored within the EMR (for current patients) and can be directly extracted to facilitate analysis. Patients on puberty blockers and gender affirming hormones are reviewed by clinicians approximately every $3-6$ months, and we will routinely extract physical health information generated from these appointments from patients' medical record. For those who no longer attend the RCHGS, this information will be sought from patients' current treating clinician, at approximately the same time as their scheduled online assessments.

\section{Sample size}

In the first 24 months of Trans20, 397 young people were considered eligible for involvement in Trans20. Assuming similar rates for the final 12 months of the 3-year enrolment period, it is expected that the total sample will comprise approximately 600 patients. Taking this into consideration, the starting sample size is large enough to estimate with $90 \%$ power a minimum difference of $6 \%$ between two proportions or a minimum difference of $0.3 \mathrm{SD}$ between two means of two equal size groups. The statistical power may not be the same for unequal or subgroup analysis.

To estimate attrition, the number of questionnaires completed before an MDAC (or 12 months post FASST) were examined relative to those who had completed questionnaires before their FASST appointment across the first 24 months of Trans20, resulting in a retention rate of $83 \%$. Based on these figures, it is anticipated that 498 young people will continue completing questionnaires post the 3-year recruitment period.

\section{Data analysis plan}

Relevant clinical outcomes (GD, mental health, physical health, schooling, family functioning and quality of life) will be described using appropriate univariate statistics and by subgroups such as gender identity, birth-assigned sex and treatment status using bivariate statistics. Multivariate models such as linear regression or logistic regression will be used for continuous or bivariate measures, respectively, to explore the relationship between physiological and clinical outcomes with gender identity adjusting for covariates. Appropriate statistical methods will be applied to analyse the repeated measures of factors over time, their effect on the outcomes including missing data if necessary.

\section{Patient and public involvement}

The RCHGS Consumer Advisory Group comprises children, adolescents and young adults who identify as TGD, families of those who identity as TGD, as well as representatives from organisations that work with or support the trans community. The RCHGS Consumer Advisory Group was consulted about the Trans20 proposal and provided input into the instruments used in Trans 20 and the preferred methods of data collection. The Consumer Advisory Group will continue to be consulted to ensure that this research is conducted in a way that best suits the needs of the trans, gender diverse and non-binary community.

\section{ETHICS AND DISSEMINATION}

The Royal Children's Hospital Human Research Ethics Committee (HREC) granted approval for the conduct of this study (\#36323).

Findings from Trans20 will have translational impact by informing future treatment guidelines and gender affirming healthcare practices. It is expected that findings from the Trans20 study will be presented at conferences and published in peer reviewed journals.

\section{DISCUSSION}

Providing optimal clinical care for TGD young people is critical, but there is a need for stronger empirical research data to inform clinical practice in this emerging field. The Trans20 study has been established to examine biopsychosocial outcomes of TGD individuals attending a large, multidisciplinary paediatric gender service. The majority of data for Trans20 are collected prospectively as part of routine clinical care to assist clinicians in providing patient care and simultaneously offer an opportunity to address important research questions in the field of transgender child and adolescent health. 
Several features of Trans20 are worth highlighting. First, the longitudinal nature of the study, spanning childhood, adolescence and young adulthood will allow a number of critical questions to be addressed. The answers to these will shed light on trajectories of gender identity development in early life, risk and protective factors related to health outcomes and the safety and effectiveness of psychosocial and hormonal interventions. Second, the collected data have significant breadth and depth, providing detailed information on gender identity, transition, dysphoria and gender-related healthcare and on mental health, physical health, education, quality of life and family functioning, which will allow for a comprehensive range of outcomes to be examined. Third, study participants are all from a single paediatric gender service that employs a consistent assessment and treatment approach and, in this way, Trans20 avoids some of the difficulties inherent in studies that recruit across multiple sites where treatment approaches may vary.

Similar to other cohort studies, the issue of attrition should be considered in light of its potential to impact the generalisability and power of the study. Notwithstanding this, as the initial enrolment into Trans20 includes all patients attending the RCHGS who complete questionnaires, there is less likelihood of selection bias occurring as part of study recruitment. Furthermore, it is not ethically possible to incorporate an untreated control group in the Trans20 study design. This is because withholding treatment for the purposes of forming a comparison group may cause patients significant distress and therefore pose significant risk of harm to individuals. Absence of a control group will limit the potential to draw direct conclusions about the effectiveness of interventions but, where possible, measures with population-based data are used in Trans20 to compare outcomes of TGD youth with those of the general population.

In conclusion, referrals of TGD children and adolescents for medical care have been increasing across the Western world, and the current demand for transgender health services may be just the tip of the iceberg. Looking ahead, it will be paramount to fill existing knowledge gaps and determine empirically how best to manage the care of TGD young people so that future best practice guidelines can be based on as much robust evidence as possible. In this regard, the Trans20 study will provide critical information pertinent to clinical practice and its provision. It will provide integral information on the natural history of gender diversity, which will enable clinicians to provide accurate prognostic information to patients and families and therefore assist decision-making around social and legal transition for TGD young people. The study will also provide important information on the benefits and risks of current clinical pathways which could be used to inform the TGD community about the long-term safety and outcomes of different forms of medical interventions available to them. Finally, the longitudinal nature of Trans20 will allow opportunities for targeted interventions to be identified and ultimately help improve care for this vulnerable population.

\section{Author affiliations}

${ }^{1}$ Murdoch Children's Research Institute, Parkville, Victoria, Australia
${ }^{2}$ Department of Adolescent Medicine, The Royal Children's Hospital Melbourne, Parkville, Victoria, Australia

${ }^{3}$ Melbourne Graduate School of Education, The University of Melbourne, Parkville, Victoria, Australia

${ }^{4}$ Department of Paediatrics, The University of Melbourne, Parkville, Victoria, Australia ${ }^{5}$ Clinical Epidemiology and Biostatistics Unit, Murdoch Children's Research Institute, Parkville, Victoria, Australia

${ }^{6}$ The Walter and Eliza Hall Institute of Medical Research, Parkville, Victoria, Australia

Acknowledgements We would like to acknowledge Associate Professor Sam Winter, Dr Riki Lane, Associate Professor Ruth McNair, Associate Professor Cathrine Mihalopoulos, Professor David Coghill as well as the RCHGS team for their intellectual input into the Trans20 study design. We would also like to thank the RCHGS Consumer Advisory Group for providing feedback about the Trans20 proposal and for providing input into the instruments used in Trans20 and the preferred methods of data collection.

Contributors KP, MAT, MT and CP conceptualised the design of the study. MH devised the analysis plan. JB, CC and NF helped to implement the study. MAT drafted the manuscript. All authors reviewed and edited the manuscript and approved the final manuscript.

Funding The Trans20 study is supported by the RCH Foundation.

Competing interests None declared.

Patient consent for publication Not required.

Provenance and peer review Not commissioned; externally peer reviewed.

Open access This is an open access article distributed in accordance with the Creative Commons Attribution Non Commercial (CC BY-NC 4.0) license, which permits others to distribute, remix, adapt, build upon this work non-commercially, and license their derivative works on different terms, provided the original work is properly cited, appropriate credit is given, any changes made indicated, and the use is non-commercial. See: http://creativecommons.org/licenses/by-nc/4.0/.

\section{ORCID iDs}

Michelle Anne Tollit https://orcid.org/0000-0003-4940-0841

Monsurul Hoq http://orcid.org/0000-0002-0504-9335

Janet Bryson http://orcid.org/0000-0001-9817-2481

Ken C Pang https://orcid.org/0000-0002-6881-775X

\section{REFERENCES}

1. Meerwijk EL, Sevelius JM. Transgender population size in the United States: a meta-regression of population-based probability samples. Am J Public Health 2017;107:e1-8.

2. Goodman M, Adams N, Corneil T, et al. Size and distribution of transgender and gender Nonconforming populations: a narrative review. Endocrinol Metab Clin North Am 2019;48:303-21.

3. Strauss P, Cook A, Winter S, et al. Trans Pathways: the mental health experiences and care pathways of trans young people. Summary of results. Perth, Australia: Telethon Kids Institute, 2017.

4. Earnshaw VA, Bogart LM, Poteat VP, et al. Bullying among lesbian, gay, bisexual, and transgender youth. Pediatr Clin North Am 2016;63:999-1010.

5. Huffaker L, Kwon P. A comprehensive approach to sexual and transgender prejudice. J Gay Lesbian Soc Serv 2016;28:195-213.

6. Winter S, Diamond M, Green J, et al. Transgender people: health at the margins of society. The Lancet 2016;388:390-400.

7. Heard J, Morris A, Kirouac N, et al. Gender dysphoria assessment and action for youth: review of health care services and experiences of trans youth in Manitoba. Paediatr Child Health 2018;23:179-84.

8. The Tavistock and Portman NHS Foundation Trust. GIDS referrals increase in 2017/18, 2018. Available: http://gids.nhs.uk/news-events/ 2018-05-16/gids-referrals-increase-201718

9. Spack NP, Edwards-Leeper L, Feldman HA, et al. Children and adolescents with gender identity disorder referred to a pediatric medical center. Pediatrics 2012;129:418-25.

10. Telfer M, Tollit M, Feldman D. Transformation of health-care and legal systems for the transgender population: the need for change in Australia. J Paediatr Child Health 2015;51:1051-3.

11. Coleman E, Bockting W, Botzer M, et al. Standards of care for the health of transsexual, transgender, and gender-nonconforming people, version 7. Int J Transgend 2012;13:165-232.

12. Hembree WC, Cohen-Kettenis PT, Gooren L, et al. Endocrine treatment of Gender-Dysphoric/Gender-Incongruent persons: an 
endocrine Society clinical practice guideline. J Clin Endocrinol Metab 2017;102:3869-903.

13. Telfer MM, Tollit MA, Pace CC, et al. Australian standards of care and treatment guidelines for transgender and gender diverse children and adolescents. Med J Aust 2018;209:132-6.

14. Marinkovic M, Newfield RS. Chest reconstructive surgeries in transmasculine youth: experience from one pediatric center. Int $J$ Transgend 2017;18:376-81.

15. Olson-Kennedy J, Warus J, Okonta V, et al. Chest reconstruction and chest dysphoria in transmasculine minors and young adults: comparisons of nonsurgical and postsurgical cohorts. JAMA Pediatr 2018;172:431-6.

16. Schuster MA, Reisner SL, Onorato SE. Beyond bathrooms-meeting the health needs of transgender people. N Engl J Med 2016;375:101-3.

17. Chew D, Anderson J, Williams $\mathrm{K}$, et al. Hormonal treatment in young people with gender dysphoria: a systematic review. Pediatrics 2018;141:e20173742.

18. Ehrensaft D. Raising Girlyboys: a parent's perspective. Studies in Gender and Sexuality 2007;8:269-302.

19. Temple Newhook J, Pyne J, Winters K, et al. A critical commentary on follow-up studies and "desistance" theories about transgender and gender-nonconforming children. Int J Transgend 2018;19:212-24.

20. Drummond KD, Bradley SJ, Peterson-Badali M, et al. A followup study of girls with gender identity disorder. Dev Psychol 2008;44:34-45.

21. Steensma TD, Biemond R, de Boer F, et al. Desisting and persisting gender dysphoria after childhood: a qualitative follow-up study. Clin Child Psychol Psychiatry 2011;16:499-516.

22. Steensma TD, McGuire JK, Kreukels BPC, et al. Factors associated with desistence and persistence of childhood gender dysphoria: a quantitative follow-up study. J Am Acad Child Adolesc Psychiatry 2013;52:582-90.

23. Wallien MSC, Cohen-Kettenis PT. Psychosexual outcome of gender-dysphoric children. J Am Acad Child Adolesc Psychiatry 2008;47:1413-23.

24. de Vries ALC, McGuire JK, Steensma TD, et al. Young adult psychological outcome after puberty suppression and gender reassignment. Pediatrics 2014;134:696-704.

25. Reardon S. Largest ever study of transgender teenagers set to kick off. Nature 2016;531:560.

26. GmbH L. LimeSurvey: an open source survey tool. Hamburg, Germany: LimeSurvey GmbH.

27. McLennan W. Standards for statistics on cultural and language diversity. Canberra: Australian Bureau of Statistics, 1999.

28. Australian Bureau of Statistics. Census household form, 2016. Available: http://www.abs.gov.au/ausstats/abs@.nsf/Lookup/2901. OMain\%20Features802016/\$FILE/2016\%20Census\%20Sample\% 20 Household\%20Form.pdf

29. Johnson LL, Bradley SJ, Birkenfeld-Adams AS, et al. A parentreport gender identity questionnaire for children. Arch Sex Behav 2004;33:105-16.

30. Hakeem A, Črnčec R, Asghari-Fard M, et al. Development and validation of a measure for assessing gender dysphoria in adults: the gender Preoccupation and stability questionnaire. Int $J$ Transgend 2016:17:131-40.

31. Lindgren TW, Pauly IB. A body image scale for evaluating transsexuals. Arch Sex Behav 1975;4:639-56.

32. Dacakis G, Davies S, Oates JM, et al. Development and preliminary evaluation of the transsexual voice questionnaire for male-to-female transsexuals. J Voice 2013;27:312-20.

33. Davies SM, Johnston JR. Exploring the validity of the transsexual voice questionnaire for male-to-female transsexuals. Can J SpeechLang Pathol Audiol;39:40-51.

34. Achenbach T, Rescorla LA. Manual for the ASEBA School-Age Forms \& Profiles. Burlington, VT: University of Vermont, Research Center for Children, Youth, \& Families, 2001.

35. Achenbach T, Rescorla LA. Manual for the ASEBA Preschool Forms \& Profiles. Burlington, VT: University of Vermont, Research Center for Children, Youth, \& Families, 2000

36. Angold A, Costello EJ, Messer SC, et al. Development of a short questionnaire for use in epidemiological studies of depression in children and adolescents. Int J Meth Psych Res 1995;5:237-49.

37. Edwards SL, Rapee RM, Kennedy SJ, et al. The assessment of anxiety symptoms in preschool-aged children: the revised preschool anxiety scale. J Clin Child Adolesc Psychol 2010;39:400-9.

38. Nauta MH, Scholing A, Rapee RM, et al. A parent-report measure of children's anxiety: psychometric properties and comparison with child-report in a clinic and normal sample. Behav Res Ther 2004;42:813-39.
39. Spence SH. A measure of anxiety symptoms among children. Behav Res Ther 1998;36:545-66.

40. Spence $\mathrm{SH}$. Structure of anxiety symptoms among children: a confirmatory factor-analytic study. J Abnorm Psychol 1997;106:280-97.

41. Spence SH, Barrett PM, Turner CM. Psychometric properties of the Spence children's anxiety scale with young adolescents. J Anxiety Disord 2003;17:605-25.

42. Lovibond SH, Lovibond PF. Manual for the depression anxiety stress scales. 2nd edn. Sydney: Psychology Foundation, 1995

43. Mattick RP, Clarke JC. Development and validation of measures of social phobia scrutiny fear and social interaction anxiety. Behav Res Ther 1998;36:455-70.

44. Testa RJ, Habarth J, Peta J, et al. Development of the gender minority stress and resilience measure. Psychology of Sexual Orientation and Gender Diversity 2015;2:65-77.

45. Smith BW, Dalen J, Wiggins K, et al. The brief resilience scale: assessing the ability to bounce back. Int J Behav Med 2008;15:194-200.

46. Hawton K, Rodham K, Evans E, et al. Deliberate self harm in adolescents: self report survey in schools in England. BMJ 2002;325:1207-11.

47. Posner K, Brown GK, Stanley B, et al. The Columbia-Suicide severity rating scale: initial validity and internal consistency findings from three multisite studies with adolescents and adults. Am J Psychiatry 2011;168:1266-77.

48. Selzer R, Hamill C, Bowes G, et al. The branched eating disorders test: validity in a nonclinical population. Int J Eat Disord 1996;20:57-64

49. Van Der Miesen AIR, Hurley H, De Vries ALC. Gender dysphoria and autism spectrum disorder: a narrative review. Int Rev Psychiatry 2016;28:70-80

50. Constantino J, Gruber C. The social responsiveness scale manual, second edition (SRS-2). Los Angeles, CA: Western Psychological Services, 2012

51. Goodenow C. The psychological sense of school membership among adolescents: scale development and educational correlates. Psychol Sch 1993;30:79-90.

52. Daraganova G. Is it OK to be away? School attendance in the primary school years. The longitudinal study of Australian children annual statistical report. Australian Institute of Family Studies, 2012.

53. Bond L, Wolfe S, Tollit M, et al. A comparison of the Gatehouse bullying scale and the peer relations questionnaire for students in secondary school. J Sch Health 2007;77:75-9.

54. Victorian Agency for Health Information. Victorian population health survey 2017. Melbourne: VAHI, 2019.

55. Stevens K. Assessing the performance of a new generic measure of health-related quality of life for children and refining it for use in health state valuation. Appl Health Econ Health Policy 2011;9:157-69.

56. Stevens $\mathrm{K}$. Valuation of the child health utility 9D index. Pharmacoeconomics 2012;30:729-47.

57. Hawthorne G, Richardson J, Osborne R. The assessment of quality of life (AQoL) instrument: a psychometric measure of health-related quality of life. Qual Life Res 1999;8:209-24

58. Richardson J, Hawthorne G. The Australian quality of life (AQoL) instrument: psychometric properties of the descriptive system and initial validation. Australian Studies in Health Service Administration, 1998: 315-42.

59. Hawthorne G, Korn S, Richardson J. Population norms for the AQoL derived from the 2007 Australian national survey of mental health and wellbeing. Aust N Z J Public Health 2013:37:7-16.

60. Furber G, Segal $L$. The validity of the child health utility instrument (CHU9D) as a routine outcome measure for use in child and adolescent mental health services. Health Qual Life Outcomes 2015;13:1.

61. Epstein NB, Baldwin LM, Bishop DS. The McMaster family assessment device. J Marital Fam Ther 1983;9:171-80.

62. Miller IW, Epstein NB, Bishop DS, et al. The McMaster family assessment device: reliability and validity*. J Marital Fam Ther 1985;11:345-56.

63. Pollard JA, Hawkins JD, Arthur MW. Risk and protection: are both necessary to understand diverse behavioral outcomes in adolescence? Soc Work Res 1999;23:145-58.

64. Glaser RR, Horn MLV, Arthur MW, et al. Measurement properties of the communities that Care youth survey across demographic groups. $J$ Quant Criminol 2005;21:73-102.

65. Arthur MW, Hawkins JD, Pollard JA, et al. Measuring risk and protective factors for substance use, delinquency, and other adolescent problem behaviors. The communities that care youth survey. Eval Rev 2002;26:575-601. 
66. Mundy LK, Simmons JG, Allen NB, et al. Study protocol: the childhood to adolescence transition study (cats). BMC Pediatr 2013;13:160.

67. Bockting W, Robinson B, Benner A, et al. Patient satisfaction with transgender health services. J Sex Marital Ther 2004;30:277-94.

68. Davies A, Bouman WP, Richards C, et al. Patient satisfaction with gender identity clinic services in the United Kingdom. Sexual and Relationship Therapy 2013;28:400-18.

69. Erasmus J, Bagga H, Harte F. Assessing patient satisfaction with a multidisciplinary gender dysphoria clinic in Melbourne. Australas Psychiatry 2015;23:158-62.
70. Wylie KR, Fitter J, Bragg A. The experience of service users with regard to satisfaction with clinical services. Sexual and Relationship Therapy 2009;24:163-74.

71. Marshall WA, Tanner JM. Variations in pattern of pubertal changes in girls. Arch Dis Child 1969;44:291-303.

72. Marshall WA, Tanner JM. Variations in the pattern of pubertal changes in boys. Arch Dis Child 1970;45:13-23.

73. Morris NM, Udry JR. Validation of a self-administered instrument to assess stage of adolescent development. $J$ Youth Adolesc 1980;9:271-80. 